\title{
Baryon spectroscopy at BESIII
}

\section{Beijiang LIU (for BESIII collaboration)*}

Institute of High Energy Physics, Chinese Academy of Sciences, 19B Yuanquanlu, Shijingshan district, Beijing, 100049, China

E-mail: Liubjeihep.ac.cn

The BESIII experiment in Beijing takes data in $\tau$-charm domain since 2009 . For the moment the world largest samples of $J / \psi, \psi(3686), \psi(3770)$ and $\psi(4040)$ data have been collected. Hadron spectroscopy is a unique way to access QCD, which is one of the most important physics goals of BESIII. Charmonium decays provide an excellent place for studying excited nucleons and hyperons $-N^{*}, \Lambda^{*}, \Sigma^{*}$ and $\Xi^{*}$ resonances. Some recent results are presented for the baryon resonance program at BES.

XV International Conference on Hadron Spectroscopy-Hadron 2013

4-8 November 2013

Nara, Japan

${ }^{*}$ Speaker. 


\section{Introduction}

Baryons are the basic building blocks of our world. Since baryons represent the simplest system in which the three colors of QCD neutralize into colorless objects and the essential nonAbelian character of QCD is manifest, understanding the baryon structure is absolutely necessary before we claim that we really understand QCD. Hadron spectroscopy is a unique way to access QCD. Given many recent processes, our present baryon spectroscopy is still in its infancy[四. Many fundamental issues in baryon spectroscopy are still not well understood[[], [3]. The possibility of new, as yet unappreciated, symmetries could be addressed with accumulation of more data. The new symmetries may not have obvious relation with QCD, just like nuclear shell model and collective motion model.

BESIII (Beijing Spectrometer) is a new state-of-the-art $4 \pi$ detector at the upgraded BEPCII (Beijing Electron and Positron Collider) that operated in the $\tau$-charm threshold energy region[团]. Since 2009, it has collected the world's largest data samples of $J / \psi, \psi(3686), \psi(3770)$ and $\psi(4040)$ decays. These data are being used to make a variety of interesting and unique studies of light hadron spectroscopy precision charmonium physics and high-statistics measurements of $\mathrm{D}$ meson decays [[5].

The $J / \psi$ and $\psi^{\prime}$ experiments at BES provide an excellent place for studying excited nucleons and hyperons $-N^{*}, \Lambda^{*}, \Sigma^{*}$ and $\Xi^{*}$ resonances[ [G]. The corresponding Feynman graph for the production of these excited nucleons and hyperons is shown in Fig. $\square$ where $\Psi$ represents either $J / \psi$ or $\psi^{\prime}$.

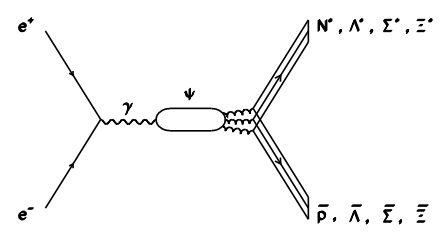

Figure 1: $\bar{p} N^{*}, \bar{\Lambda} \Lambda^{*}, \bar{\Sigma} \Sigma^{*}$ and $\bar{\Xi} \Xi^{*}$ production from $e^{+} e^{-}$collision through $\Psi$ meson.

Complementary to other facilities, the baryon program at BES3 has several advantages [ $[$ ]. For instance, $\pi N$ and $\pi \pi N$ systems from $J / \psi \rightarrow \bar{N} N \pi$ and $\bar{N} N \pi \pi$ processes have an isospin of $1 / 2$ due to isospin conservation; $\psi$ mesons decay to baryon-antibaryon pairs through three or more gluons, where is a favorable place for producing hybrid (qqqg) baryons, and for searching some "missing" $N^{*}$ resonances which have weak coupling to both $\pi N$ and $\gamma N$, but stronger coupling to $g^{3} N$.

\section{Partial Wave Analysis of $\psi(3686) \rightarrow p \bar{p} \eta[$ [Q]}

In this analysis, the $\eta$ is constructed via its decay into two photons. Figure $\square$ shows the scatter plot of $M_{p \bar{p}}$ versus $M_{\gamma \gamma}$ for events after initial event selections, where the two vertical bands correspond to the decays $\psi(3686) \rightarrow p \bar{p} \pi^{0}$ and $\psi(3686) \rightarrow p \bar{p} \eta$, and the horizontal band corresponds to the decay $\psi(3686) \rightarrow X+J / \psi(J / \psi \rightarrow p \bar{p})$.

Fig. B] shows the PWA results, including the invariant mass spectra of $p \bar{p}, \eta p, \eta \bar{p}$ and angular distributions, which are consistent with the data. The solution indicates that $N(1535)$ combined 


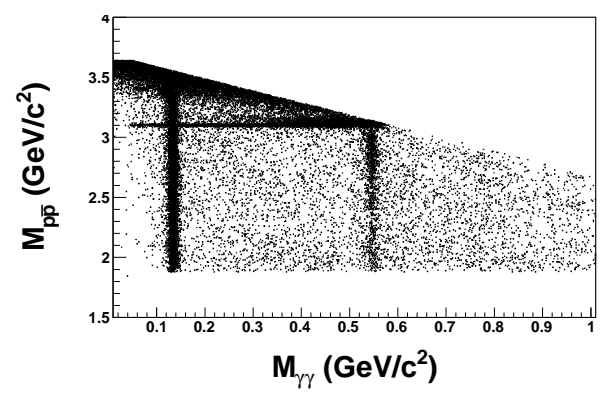

Figure 2: Scatter plots of $p \bar{p}$ invariant mass versus $\gamma \gamma$ invariant mass

with an interfering non-resonant contribution is sufficient to describe the data. We observe $527 \pm 27$ $N(1535) \rightarrow p \eta$ events with a mass $M=1524 \pm 5_{-4}^{+10} \mathrm{MeV} / c^{2}$, a width $\Gamma=130_{-24-10}^{+27+57} \mathrm{MeV} / c^{2}$, and a statistical significance larger than $10 \sigma$. The product branching fraction of $\psi(2 S) \rightarrow N(1535) \bar{p}$ $(N(1535) \rightarrow p \eta)$ is calculated to be $\left(5.2 \pm 0.3_{-1.2}^{+3.2}\right) \times 10^{-5}$. Here, the first error is statistical and the second systematic.

To investigate the $p \bar{p}$ mass enhancement, a scan for an additional $1^{--}$resonance described by a Breit-Wigner function is performed. There is no evidence for a $p \bar{p}$ resonance in this region, indicating that the threshold enhancement can be explained by interference between the $N(1535)$ and phase space.
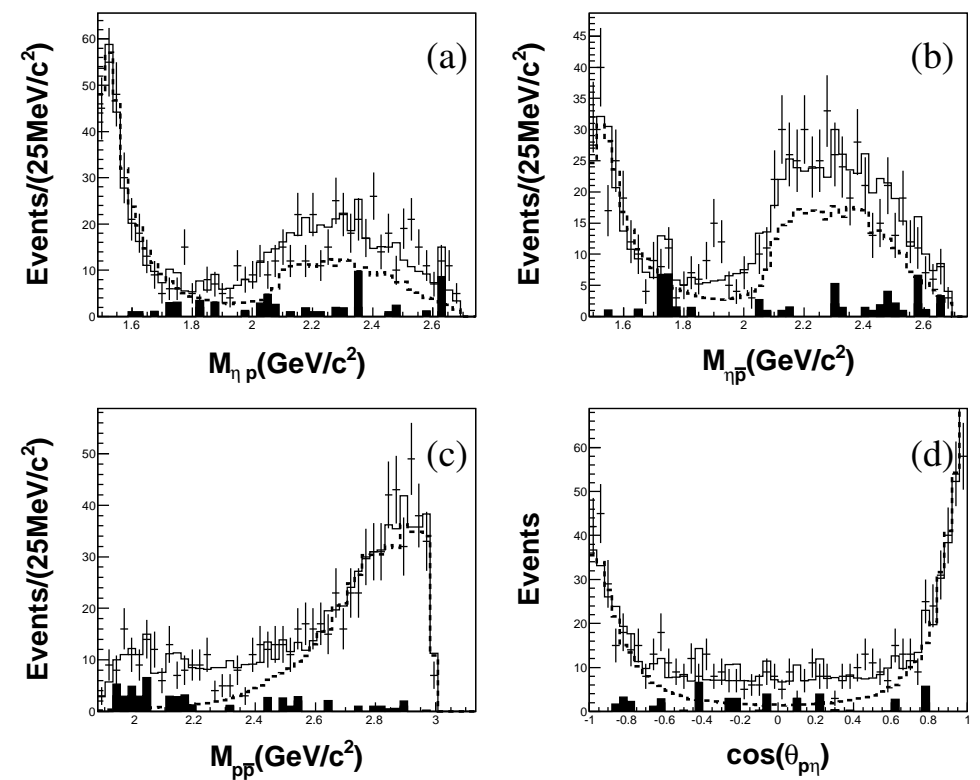

Figure 3: Distributions of (a) $M_{p \eta}$, (b) $M_{\bar{p} \eta}$, (c) $M_{p \bar{p}}$ and (d) the angle between $p \eta$ in the $p \bar{p}$ system. The crosses are for data, the blank histograms for PWA projections, the dashed lines for the contribution of $N(1535)$ and the shaded histograms for the background events from $\eta$ sidebands and continuum data.

\section{Partial Wave Analysis of $\psi(3686) \rightarrow p \bar{p} \pi^{0}[\mathbf{8}]$}

The Dalitz plot and the invariant mass of $p \bar{p}$ of this decay are shown in Fig. T(a) and (b) and the invariant mass of $p \pi^{0}$ and $\bar{p} \pi^{0}$ are shown in plots(c) and (d) of Fig. 田 respectively, in which some $N^{*}$ states can been seen. 
In the PWA of this decay, $19 N^{*}$ resonances and a hypothetical $p \bar{p}$ resonance are considered. The significance of each resonance is determined. Resonances with significance greater than $5 \sigma$ are taken as significant ones which include $N(940)$ and seven $N^{*}$ resonances. Table $\square$ lists the optimized values for the seven $N^{*}$ states. In this table, the first five $N^{*}$ resonances are consistent with the values in the Particle Data Book [四, while the last two are new. The significance of these two states are $15 \sigma$ and $11.7 \sigma$, respectively. The names of these two new states are labeled as $N(2300)$ and $N(2570)$ according to the optimized masses, with $J^{P}$ assignment of $1 / 2^{+}$and $5 / 2^{-}$, respectively.

Table 1: The optimized mass, width and significance (Sig.) of the seven significant $N^{*}$ resonances. $\Delta S$ represents the change of the log likelihood value. $\Delta N_{\text {dof }}$ is the change of the number of free parameters in the fit. In the second and third columns, the first error is statistical and the second is systematic.

\begin{tabular}{cccccc} 
Resonance & $\mathrm{M}\left(\mathrm{MeV} / c^{2}\right)$ & $\Gamma\left(\mathrm{MeV} / c^{2}\right)$ & $\Delta S$ & $\Delta N_{d o f}$ & Sig. \\
\hline$N(1440)$ & $1390_{-21-30}^{+11+21}$ & $340_{-40-156}^{+46+70}$ & 72.5 & 4 & $11.5 \sigma$ \\
$N(1520)$ & $1510_{-7-9}^{+3+11}$ & $115_{-15-40}^{+20+0}$ & 19.8 & 6 & $5.0 \sigma$ \\
$N(1535)$ & $1535_{-8-22}^{+9+15}$ & $120_{-20-42}^{+20+0}$ & 49.4 & 4 & $9.3 \sigma$ \\
$N(1650)$ & $1650_{-5-30}^{+5+11}$ & $150_{-22-50}^{+21+14}$ & 82.1 & 4 & $12.2 \sigma$ \\
$N(1720)$ & $1700_{-28-35}^{+30+32}$ & $450_{-94}^{+109+149}$ & 55.6 & 6 & $9.6 \sigma$ \\
$N(2300)$ & $2300_{-30-0}^{+40+109}$ & $340_{-30-58}^{+30+110}$ & 120.7 & 4 & $15.0 \sigma$ \\
$N(2570)$ & $2570_{-10-10}^{+19+34}$ & $250_{-24-21}^{+14+69}$ & 78.9 & 6 & $11.7 \sigma$
\end{tabular}

Using these eight significant resonances, the fit result agrees well with the data, as shown in Fig. W. On the basis of the eight significant states, a scan for additional resonances has been performed with different spin parity, mass and width combinations. No extra resonance has been found to be significant. Besides the known and speculative $N^{*}$ resonances, a $1^{--} p \bar{p}$ resonance candidate described by the Breit-Wigner function has been added, as suggested by the near threshold enhancement in the $p \bar{p}$ mass distribution. The largest significance obtained is $4 \sigma$ at a mass of $2000 \mathrm{MeV} / c^{2}$ and width of $50 \mathrm{MeV} / c^{2}$, indicating that no $p \bar{p}$ resonance is required to explain the threshold enhancement.

\section{Summary}

Based on $106 \times 10^{6} \psi(3686)$ events collected with the BESIII at BEPCII, partial wave analyses (PWA) of $\psi(3686) \rightarrow p \bar{p} \eta$ and $\psi(3686) \rightarrow p \bar{p} \pi^{0}$ are performed. Two new $N^{*}$ resonances are observed in $\psi(3686) \rightarrow p \bar{p} \pi^{0}$ decays, one is $\mathrm{N}(2300)(1 / 2+)$ with a mass of $2300_{-30-0}^{+40+109} \mathrm{MeV} / c^{2}$ and width of $340_{-30-58}^{+30+110} \mathrm{MeV} / c^{2}$, and one is $\mathrm{N}(2570)(5 / 2-)$ with a mass of $2570_{-10-10}^{+19+34} \mathrm{MeV} / c^{2}$ and width of $250_{-24-21}^{+14+69} \mathrm{MeV} / c^{2}$.

Now five years from our first collisions, BESIII has established a broad and successful program in charm physics. Recently, in 2012, even larger samples have been accumulated at the $J / \psi$ and $\psi(3686)$; total samples are now about 1.2 billion and 0.35 billion decays, respectively. Furthermore, our 2013 dataset includes more data near $4260 \mathrm{MeV}$, and also a large sample at the $Y(4360)$. With the excellent performance of the accelerator and detector, more interesting results are expected. 

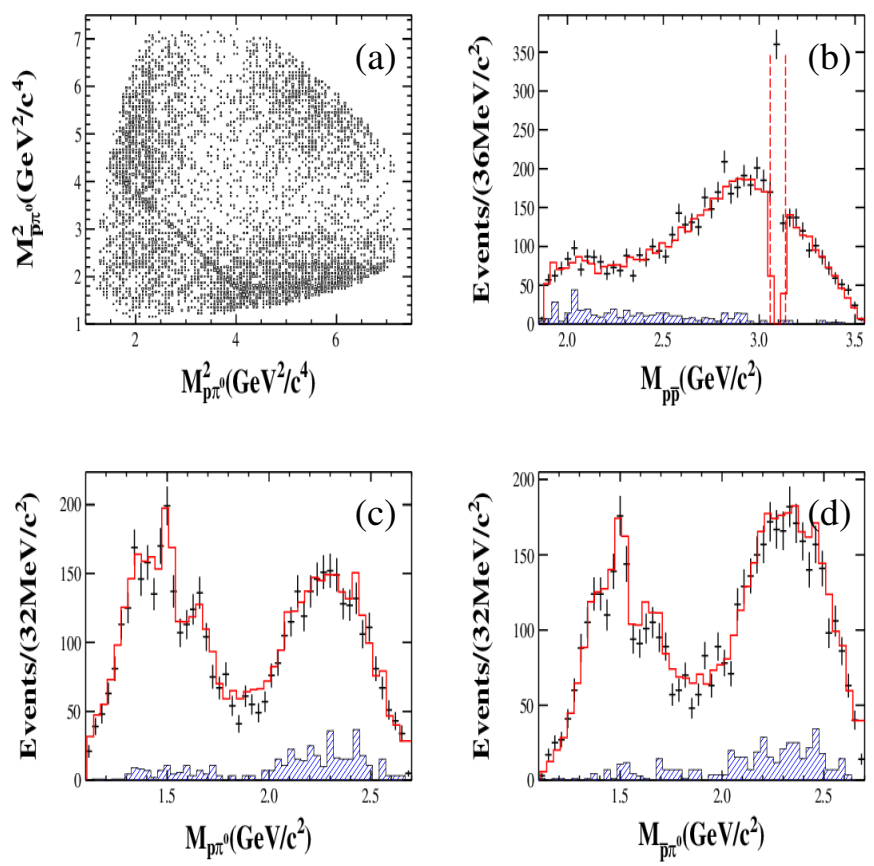

Figure 4: (a) The Dalitz plot of $\psi(3686) \rightarrow p \bar{p} \pi^{0}$, the invariant mass spectra of (b) $p \bar{p}$, (c) $p \pi^{0}$, and (d) $\bar{p} \pi^{0}$. The dashed lines in (b) show the cut at the $J / \psi$ mass region. The crosses represent the experimental data, and the shaded histograms show the background from continuum process and $\pi^{0}$ sideband. The histograms in solid line show the sum of MC prediction and the background.

\section{References}

[1] J. Beringer et al. [Particle Data Group Collaboration], Review of Particle Physics (RPP), Phys. Rev. D 86 (2012) 010001.

[2] S. Capstick, S. Dytman, R. Holt, X. -d. Ji, J. W. Negele, E. Swanson, P. Barnes and T. Barnes et al., Key issues in hadronic physics, [hep-ph/0012238].

[3] E. Klempt and J. -M. Richard, Baryon spectroscopy, Rev. Mod. Phys. 82 (2010) 1095 [arXiv:0901.2055 [hep-ph]].

[4] M. Ablikim et al. (BESIII Collaboration), Design and Construction of the BESIII Detector, Nucl. Instrum. Meth. A 614, 345 (2010).

[5] Edited by Kuang-Ta Chao and Yi-Fang Wang, Physics at BES-III, Int. J. Mod. Phys. A 24, S1 (2009)

[6] B. -S. Zou, $N^{*}$, Lambda*, Sigma* and Xi* resonances from J / Psi and Psi-prime decays, Nucl. Phys. A 684 (2001) 330 [hep-ph/ 0006039 ].

[7] B. S. Zou et al. [BES Collaboration], Baryon spectroscopy at Beijing Electron Positron Collider, PiN Newslett. 16 (2002) 174 [hep-ph/0110264].

[8] M. Ablikim et al. [BESIII Collaboration], Observation of two new $N^{*}$ resonances in $\psi(3686) \rightarrow p \bar{p} \pi^{0}$, Phys. Rev. Lett. 110 (2013) 022001 [arXiv:1207.0223 [hep-ex]].

[9] M. Ablikim et al. [BESIII Collaboration], Partial wave analysis of $\psi(2 S) \rightarrow p \bar{p} \eta$, Phys. Rev. D 88 (2013) 032010 [arXiv: 1304.1973 [hep-ex]]. 